\title{
High-Performance Low-Loss Silicon-on-Insulator Microring Resonators using TM-polarized Light
}

\author{
P. De Heyn, B. Kuyken, D. Vermeulen, W. Bogaerts and D. Van Thourhout \\ Department of Information Technology (INTEC) - Photonics Research Group, \\ Ghent University - imec, Sint-Pietersnieuwstraat 41, B-9000 Ghent, Belgium \\ Peter.DeHeyn@intec.ugent.be
}

\begin{abstract}
Microring resonators on SOI are investigated for both orthogonal polarizations. By demonstrating low-loss $(1.94 \mathrm{~dB} / \mathrm{cm})$ microring resonators with an intrinsic Q up to 340000 we proof that using TM-polarized light enables high-performance filters.

OCIS codes: (230.0230) Optical devices; (230.5750) Resonators
\end{abstract}

\section{Introduction}

Silicon-on-Insulator microring resonators (MRR) have been demonstrated to be attractive building blocks for a wide range of photonic integrated devices due to their unique properties such as a high quality factor $(\mathrm{Q})$ and small footprint [1]. Most applications require high $\mathrm{Q}$ factors e.g. in nonlinear optics threshold power goes down with $\mathrm{Q}^{2}$ and in sensing applications a high $\mathrm{Q}$ factor increases the sensitivity. Telecom applications need to reach certain filter specifications regarding drop bandwidth and/or roll-off. All these applications have in common that they demand a high intrinsic $\mathrm{Q}\left(\mathrm{Q}_{\mathrm{i}}\right)$ representing minimal losses of the MRR itself. This high $\mathrm{Q}_{\mathrm{i}}$ will result in a high $\mathrm{Q}$ at critical coupling $\left(\mathrm{Q}_{\mathrm{c}}=\mathrm{Q}_{\mathrm{i}} / 2\right)$ as well as minimized insertion loss for (higher-order) MRR delay-lines and add-drop filters. Despite the well-established development towards low-loss deeply etched SOI waveguides using DUV lithography ( $<2 \mathrm{~dB} / \mathrm{cm}$ for TE-polarized light [2]), only moderate $\mathrm{Q}$ factors in the order of $10^{4}$ have been achieved, equivalent to an effective loss of $\sim 10 \mathrm{~dB} / \mathrm{cm}$. A part of this loss is due to backscattering on the sidewall roughness of the SOI waveguides, coherently exciting the counter-propagating mode of the MRR [3]. This effect significantly lowers the $\mathrm{Q}$ factor and causes splitting of the resonance. The degree and occurrence can be predicted by an analytical model based on the periodicity and amplitude of sidewall roughness [4] but is in principle unpredictable and for many applications highly unwanted. In this paper we show experimentally that by using the TM-polarized light instead of the more commonly used TE-polarized light, backscattering is greatly reduced, showing a higher $\mathrm{Q}_{i}$ and more predictable spectra.

\section{Theory and design}

Due to the nature of the lithography fabrication process, roughness on the vertical sidewalls of the waveguide is unavoidable. However, by using the TM polarization, the effect of this roughness can be reduced by approximately a factor 35 using standard SOI waveguides [5]. This is the result of two effects. First, the electrical field of the TM mode is pointing mainly in the vertical direction, so no discontinuity is created on the vertical sidewalls. Secondly, the TM mode is less confined resulting in a smaller modal overlap with the vertical sidewalls.

The total loss in a MRR is usually a combination of different contributions: even with perfect fabrication technology, there will be bending losses and mode-conversion loss in the coupling section and at the straight/bend interfaces of racetrack MRRs [6]. On top of that come the losses induced by scattering and backscattering at the roughness of the sidewalls. To reduce the bend losses and thereby simplifying the analysis, we designed circular MRRs with a relatively large radius $(\mathrm{R}=20 \mu \mathrm{m})$ and with symmetric directional couplers. Because the $\mathrm{TM}$ mode is less confined in a standard waveguide of $500 \mathrm{~nm} \times 220 \mathrm{~nm}$, we can expect larger coupling and larger bending losses than for the TE mode. To ensure the guidance of the TM mode close to cut-off regime, we provide an extra oxide layer as waveguide top cladding.

\section{Experimental results and discussion}

To couple light into the chip 1-dimensional near vertical grating couplers are used, designed for either TE- or TMpolarized light. Coupling from the fundamental TM mode to higher-order TE modes in adiabatic taper sections is avoided by using focusing grating couplers [7]. To make a reasonable comparison between TE and TM polarization, we show a spectrum of an add-drop MRR with the same radius $(\mathrm{R}=20 \mu \mathrm{m})$ and coupling topography, but with a larger gap for TM $(1 \mu \mathrm{m})$ than for TE $(400 \mathrm{~nm})$ providing a similar coupling strength for both polarizations. Insertion loss and crosstalk are used to describe the properties of the resonance peak, which is commonly used for filter 
specifications in telecom applications. Insertion loss (IL) is defined as the maximum of the peak at the drop port and crosstalk (XT) is defined as the difference in transmission between the peak and $0.1 \mathrm{~nm}$ off-peak.

In figure 1, the resulting resonances of this MRR using TM-polarized light are illustrated, together with their properties. From this figure, it can be seen that IL and XT are nearly constant over the whole wavelength range.

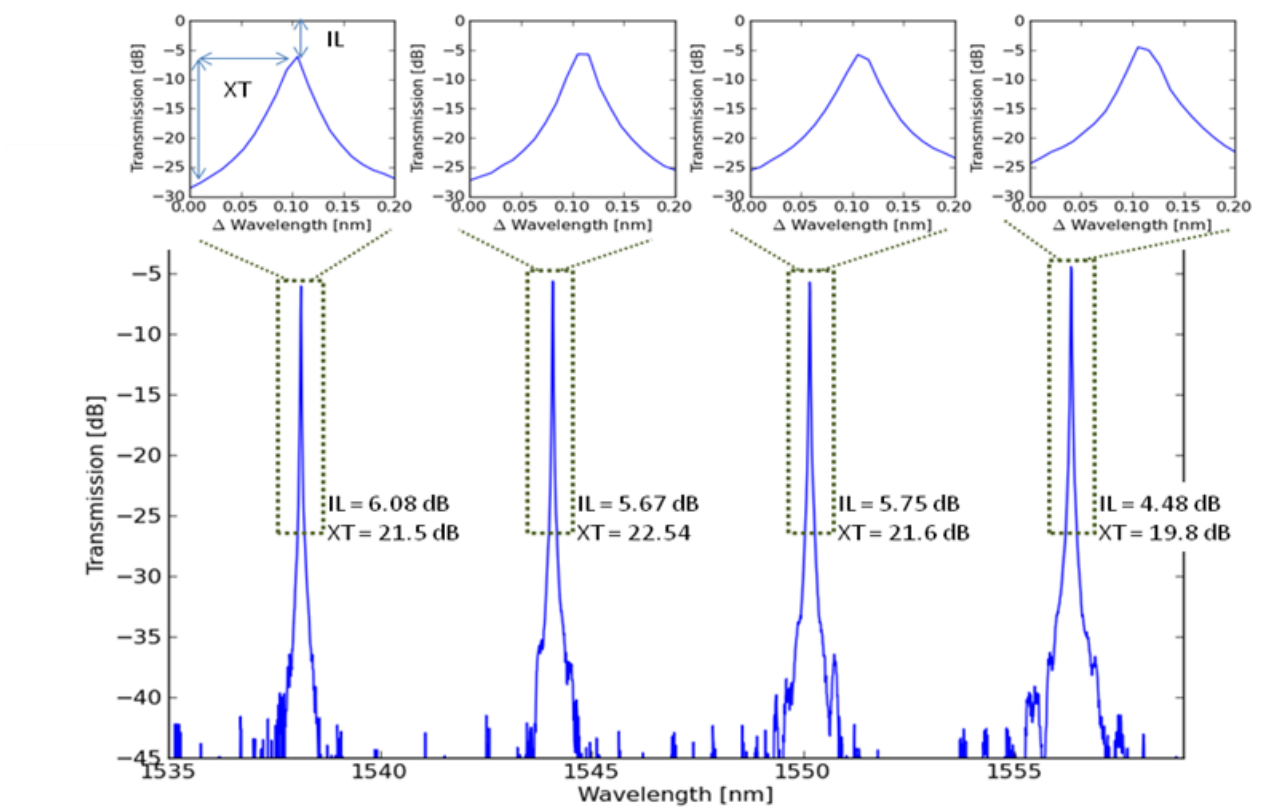

Figure 1: Transmissions spectrum taken at the drop port of an add-drop MRR $(\mathrm{R}=20 \mu \mathrm{m}$ and gap $1 \mu \mathrm{m})$ using TM-polarized light, illustrated with the nearly constant insertion loss (IL) and crosstalk (XT), showing the stable behavior of the subsequent resonances.

To demonstrate the unwanted wavelength dependency of the relative influence of backscattering using TEpolarized light, we examine the spectrum at the drop port using TE-polarized light, for a MRR with similar coupling as described earlier. This is illustrated in figure 2, where it can be clearly seen that some peaks show splitting, which degrades the insertion loss by up to $4.2 \mathrm{~dB}$ and crosstalk up to $5 \mathrm{~dB}$ with respect to peaks with less backscattering.

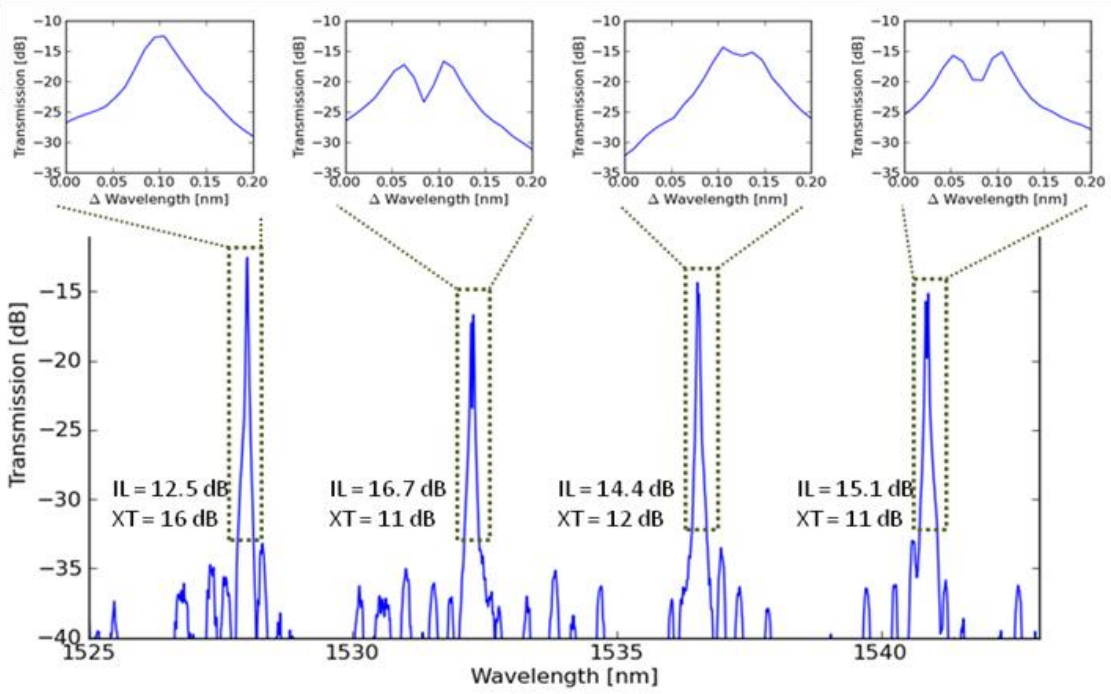

Figure 2: Transmissions spectrum taken at the drop port of an add-drop MRR $(R=20 \mu \mathrm{m}$ and gap $400 \mathrm{~nm})$ using TE-polarized light, illustrated with the insertion loss (IL) and crosstalk (XT), showing splitting of the resonances for some peaks and exhibiting unstable IL and XT.

For the resonances of the MRR using TM polarization, no peak splitting is visible, which is directly reflected on the less varying properties such as IL and XT of the TM add-drop filter. Moreover, we notice a considerable improvement for these properties using TM-polarized light instead of TE, of minimum 6dB less IL 
together with $6 \mathrm{~dB}$ more XT. To generalize these improvements for any gap, we have fabricated a whole series of MRR to measure the intrinsic loss $\left(\mathrm{Q}_{\mathrm{i}}\right)$ of the MRR. We measure the $\mathrm{Q}$ for different resonances of an all-pass filter with a gap of $1.4 \mu \mathrm{m}$ and this for a varying radius of 15,20 and $30 \mu \mathrm{m}$. For these measurements we used a high resolution of $1 \mathrm{pm}$ and the results are listed in table 1 . We assume that the coupling with this relative large gap is so small that measured $Q$ factor is close to the $Q_{i}$, from where we can extract the loss $[\mathrm{dB} / \mathrm{cm}]=4.3 \frac{2 \pi n_{g}}{\lambda_{[\mathrm{cm}]} Q_{\mathrm{t}}}$ with $n_{\mathrm{g}}$ the group index of TM- polarized light $\left(\mathrm{n}_{\mathrm{g}}=3.8\right)$ and $\lambda_{[\mathrm{cm}]}$ the wavelength expressed in $\mathrm{cm}$.

Table 1: $\mathrm{Q}_{\mathrm{i}}$, loss $[\mathrm{dB} / \mathrm{cm}]$ and extinction ratio $(\mathrm{ER})[\mathrm{dB}]$ extracted from resonances of all-pass filters with varying radius using TM-polarized light over a wavelength range between 1530-1570nm. Ranges in the table are corresponding to this wavelength range.

\begin{tabular}{|l|l|l|l|}
\hline$R[\mu \mathrm{m}]$ & $15(F S R=8 \mathrm{~nm})$ & $20(F S R=6 \mathrm{~nm})$ & $30(F S R=4 \mathrm{~nm})$ \\
\hline$Q i$ & $130000-100000$ & $160000-130000$ & $340000-270000$ \\
\hline Loss $[\mathrm{dB} / \mathrm{cm}]$ & $5.0-6.6$ & $4.1-5.0$ & $1.94-2.4$ \\
\hline$E R[\mathrm{~dB}]$ & 1.0 & $0.7-1.4$ & $0.7-1.4$ \\
\hline
\end{tabular}

From table 1, we learn that the loss decreases for increasing radius, indicating that bending losses are still present for the smaller radii. The given ranges in table 1 for $Q_{i}$ and loss are corresponding to the wavelength range between 1530-1570nm at which we examined the resonances. It is noticed that longer wavelengths seem to exhibit more loss but this cannot be unambiguously proven since one also expects a slightly larger coupling strength and hence larger coupling losses for longer wavelengths. For this purpose, we also include the extinction ration (ER) in table 1, defined as the height of the peak, where the given range corresponds again to the same wavelength range. For radii $>15 \mu \mathrm{m}$, resonance peaks at longer wavelengths exhibit a higher extinction ratio, proving that for longer wavelengths the coupling increases faster than the bending losses. Although we did not fabricate MRR with gap larger than $1.4 \mu \mathrm{m}$, it is safe to say that the $Q_{i}$ is equal or larger than 340000 , which corresponds to a loss of $1.94 \mathrm{~dB} / \mathrm{cm}$. For radius of $15 \mu \mathrm{m}$, increasing bending losses and increased coupling seem to compensate each other, resulting in a constant ER.

\section{Conclusion}

In this work we have investigated the use of TM-polarized light in add-drop and all-pass SOI MRR configurations. These resonators exhibit superior characteristics regarding insertion loss and crosstalk compared to the ones designed for TE-polarized light, both fabricated by standard DUV lithography. We demonstrate a high $\mathrm{Q}_{\mathrm{i}}$ of equal or more than 340000, roughly 4 times larger than commonly obtained using TE-polarized light. We assign these superior characteristics to the lower interaction of the TM mode with the sidewall roughness of a SOI waveguide such that backscattering is greatly reduced. Moreover, the low effective refractive index of the TM mode increases both the coupling and MRR fabrication tolerances, making the spectral behavior more stable and predictable over a wide wavelength range. This improvement enables better performance in all kind of applications ranging from lower power threshold for non-linear optics, better sensitivity for bio-sensing and lower insertion loss for (higher-order) delay-lines and filters meeting the same bandwidth specifications.

\section{Acknowlegment}

This work is partially supported by the EU project ICT-BOOM

\section{References}

[1] B.G. Lee, et. al.,"High-Performance Modulators and Switches for Silicon Photonic Networks-on-Chip," IEEE STQE, 16(1), 2010.

[2] S. Selvaraja, et. al.,"Record Low-Loss Hybrid Rib/Wire waveguides for Silicon Photonic Circuits",Group IV Photonics 2010 (PD), China, 2010

[3] B. E. Little, et. al.,"Surface-roughness-induced contradirectional coupling in ring and disk resonators", Opt. Lett.,22 (1), pp. 4-6, 1997.

[4] T. Wang, et. al.,"'Modeling of quasi-grating sidewall corrugation in SOI microring add-drop filters", Optics Communications, $282(17), 2009$.

[5] T. Barwicz, et. al.,’Three-Dimensional Analysis of Scattering Losses Due to Sidewall Roughness in Microphotonic Waveguides”, JLT, 23(9), 2005

[6] M. Soltani, et. al.,"Systematic Engineering of Waveguide-Resonator Coupling for Silicon Microring/Microdisk/Racetrack Resonators: Theory and Experiment",IEEE Journal of Quantum Electronics, 46(8), 2010.

[7] D. Vermeulen, et. al.," Efficient Tapering to the Fundamental Quasi-TM Mode in Asymmetrical Waveguides”, ECIO $2010,2010$. 